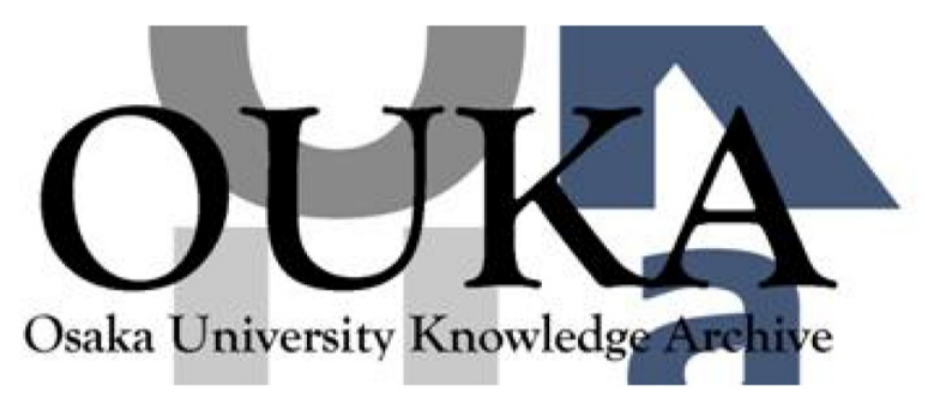

\begin{tabular}{|c|l|}
\hline Title & $\begin{array}{l}\text { Multi-imaging x-ray streak camera for } \\
\text { ultrahigh-speed two dimensional x-ray imaging } \\
\text { of imploded core plasmas(invited) }\end{array}$ \\
\hline Author(s) & $\begin{array}{l}\text { Shiraga, H.; Fujioka, S. ; Jaanimagi, P.A. et } \\
\text { al. }\end{array}$ \\
\hline Citation & $\begin{array}{l}\text { Review of Scientific Instruments. 75(10) } \\
\text { p. 3921-p.3925 }\end{array}$ \\
\hline Issue Date & 2004-10 \\
\hline oaire:version VoR \\
\hline URL & https://hdl. handle. net/11094/3471 \\
\hline rights & \\
\hline Note & \\
\hline
\end{tabular}

Osaka University Knowledge Archive : OUKA

https://ir. Library. osaka-u. ac. jp/

Osaka University 


\title{
Multi-imaging $x$-ray streak camera for ultrahigh-speed two-dimensional $x$-ray imaging of imploded core plasmas (invited)
}

\author{
H. Shiraga ${ }^{a)}$ and S. Fujioka \\ Institute of Laser Engineering, Osaka University, Suiita, Osaka 565-0871, Japan \\ P. A. Jaanimagi and C. Stoeckl \\ Laboratory for Laser Energetics, University of Rochester, Rochester, New York 14623-1299 \\ R. B. Stephens \\ General Atomics, San Diego, California 92186 \\ H. Nagatomo, K. A. Tanaka, R. Kodama, and H. Azechi \\ Institute of Laser Engineering, Osaka University, Suiita, Osaka 565-0871, Japan
}

(Presented on 21 April 2004; published 12 October 2004)

\begin{abstract}
Ultrahigh-speed two-dimensional x-ray imaging by using an image sampling technique on an $\mathrm{x}$-ray streak camera has been developed and applied to observe the imploded core plasmas of inertial confinement fusion targets. Various one-dimensional and two-dimensional sampling techniques of two-dimensional images were compared, and the multi-imaging X-ray streak camera (MIXS), was found to be one of the most successful schemes. MIXS was used in an implosion experiment of a shell target with a cone for fast ignition at the OMEGA laser facility. Nonuniform structure in imploded core plasma dynamics was clearly observed with temporal and spatial resolutions of $10 \mathrm{ps}$ and 10-20 $\mu \mathrm{m}$, respectively. (C) 2004 American Institute of Physics. [DOI: 10.1063/1.1789249]
\end{abstract}

\section{INTRODUCTION}

Ultrafast two-dimensional (2D) x-ray imaging is one of the most important plasma diagnostics for investigation of inertial confinement fusion (ICF). ${ }^{1}$ Typical imploded core plasma has a very small size and short life such as $100 \mu \mathrm{m}$ and $100 \mathrm{ps}$, respectively. Thus the required resolutions for such time-resolved imaging are of the order of $10 \mu \mathrm{m}$ and $10 \mathrm{ps}$. The ultimate goal for the resolution in diagnosing ICF plasmas at ignition would be on the order of a few ps and a few $\mu \mathrm{m}$ simultaneously.

Gated x-ray framing cameras with typical temporal resolution of $35-80$ ps coupled with imaging devices such as $\mathrm{x}$-ray pinhole cameras or $\mathrm{x}$-ray microscopes ${ }^{2-4}$ have been used for this purpose. Although these instruments work very well for the observation of the imploding shell, it is not fast enough for core plasma.

On the other hand, the multi-imaging x-ray streak camera (MIXS), which employs a one-dimensional (1D) imagesampling procedure on X-ray streak cameras, ${ }^{5-7}$ has been developed as a new ultrafast $2 \mathrm{D} x$-ray imaging technique, and has been successfully utilized for diagnosing the imploded core plasmas. ${ }^{6,7}$ It is relatively easy to obtain temporal resolution of $10 \mathrm{ps}$ or better, and even $1 \mathrm{ps}$ is possible with commercially available x-ray streak cameras. Recently, a scheme called "fast ignition (FI)" density implosion of the main fuel is accomplished by a long pulse (ns) drive laser and the heating of it by an additional ultrashort and ultraintense laser. In particular, a cone-in-shell target with a cone structure to facilitate the entrance of the

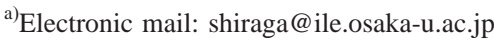

heating laser beam has been intensively studied. ${ }^{10,11}$ In such targets, the core structure and its dynamics are no longer in a spherical symmetry. It is of great importance to observe the dynamical behavior of such nonuniformly imploded core plasmas. MIXS is suitable for such 2D observation of rapidly changing phenomena.

In this article, we describe principles of image-sampling technique on streak cameras including both 1D sampling and $2 \mathrm{D}$ sampling of 2D images, as well as recent results of FI target implosion observed with MIXS.

\section{IMAGE SAMPLING TECHNIQUE ON STREAK CAMERAS}

Only x-ray streak cameras have the capability of achieving a time resolution of $10 \mathrm{ps}$ or better. Usually it is impossible to obtain time-resolved 2D images with a streak camera because a streak camera is used with a timing slit onto which only 1D image can be made as shown in Fig. 1(a). However, 2D imaging becomes possible with streak cameras if the dimensions of the image are decreased. There are two schemes to convert a $2 \mathrm{D}$ image into a set of image pieces under certain rules. Such a procedure of signal conversion is called "sampling." One of the image-sampling schemes is to convert a $2 \mathrm{D}$ image into a set of $1 \mathrm{D}$ (for example, in the $x$ direction) images by sampling the image in another direction $(y)$, just like a raster scan in a television image, as shown in Fig. 1(b). Another scheme is to convert a 2D image into a set of $(x, y)$ distributed dot signals, just as in a charge coupled device (CCD) as shown in Fig. 1(c). According to the sampling theory, the sampling interval in these configurations should be less than half of the resolution to cover the whole spatial frequencies of the image. 


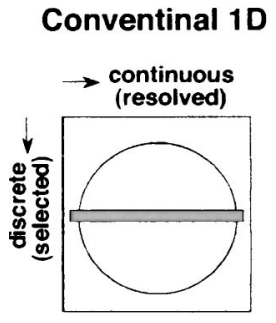

1-D selected image

(a)

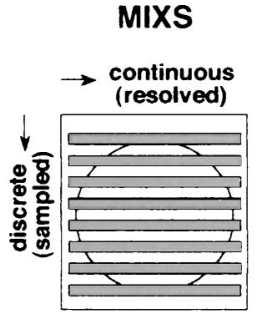

1-D image sampling

(b)

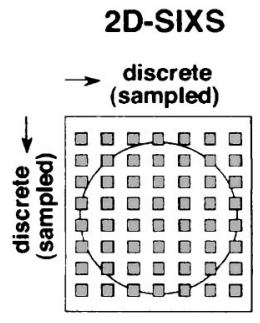

2-D image sampling

(c)
FIG. 1. Principle of image-sampling procedure applied to a streak camera: (a) conventional use of a streak camera to obtain 1D streaked images, (b) 1D sampling (MIXS scheme) to obtain 2D streaked images, and (c) 2D sampling (2D-SIXS scheme) to obtain 2D streaked images.

Image sampling on streak cameras has a long history of development for years in order to obtain time-resolved 2D images including 1D image sampling and 2D image sampling procedures. In any case, some kind of tricks must be taken into account to avoid overlapping of the signals with each other when those are streaked in time in one direction on the image.

\section{A. 1D Image sampling}

The first achievement of time-resolved 2D images with a streak camera was produced by Sultanoff in $1950 .{ }^{12}$ A grid mask similar to Fig. 1(b) was mounted on an optical camera with a fast rotating mirror. By moving the image sampled by the gird perpendicularly to the grid lines by the rotating mirror, temporal changes of the 1D images are continuously recorded between the initial positions of the neighboring grids on the film. Temporal resolution or exposure time per frame was $10 \mathrm{~ns}$, which corresponds to $10^{8}$ frames per second (fps), and 30 sequential frames were reconstructed by reversing the procedure, i.e., moving an identical grid on the recorded image on the film.

Sultanoff used only one image for 1D sampling. On the other hand, sampling can be done on repetitive signals like in a sampling oscilloscope. Repetitive gates with a slightly different frequency can sample the information from the signal into an array of discrete data. Similar procedures can be used on a 2D signal. This configuration was first demonstrated by Landen in 1992. ${ }^{5}$ Seven identical images of $\mathrm{x}$ rays emitted from a laser-irradiated foil target were made by using an arrayed pinhole camera on a cathode slit of an x-ray streak camera with a constant separation inbetween. After streaking the camera, recorded data were divided into pieces and reconstructed to from the original 2Dconfiguration. Framing speed was $3 \times 10^{10} \mathrm{fps}$ ( $30 \mathrm{ps}$ resolution).

The first observation of imploded core plasma by image sampling on a streak camera was performed in 1994 by Shiraga et al. using a MIXS by making ten identical images aligned slightly tilted on the slit of an x-ray streak camera as shown in Fig. 2(a). After streaking the camera [Fig. 2(b)], more than 50 continuously time-resolved 2D x-ray images of the imploded core plasma were reconstructed as shown in (a)
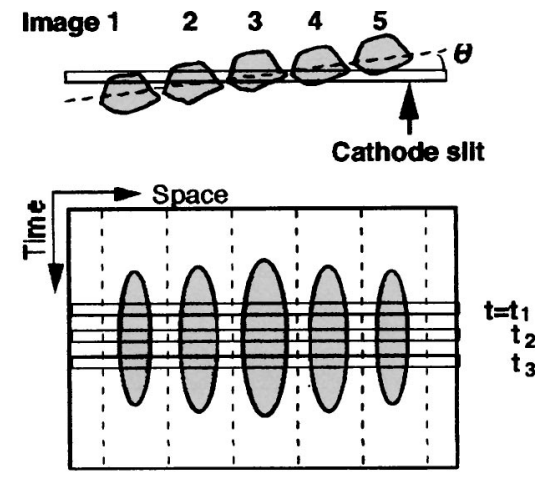

(c)

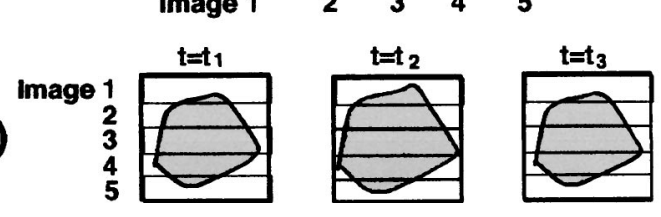

FIG. 2. Principle of MIXS imaging: (a) image array aligned on the photocathode slit, (b) streaked image array, and (c) reconstructed time-resolved images.

Fig. 2(c). ${ }^{6}$ Time and space resolutions were $10 \mathrm{ps}$ and 10-20 $\mu \mathrm{m}$, respectively. This speed corresponds to $10^{11} \mathrm{fps}$.

Variations of MIXS have been developed to improve its diagnostic capabilities. For example, a conventional 1D imaging streak scheme was coupled with 2D imaging by MIXS on the same X-ray streak camera to observe the implosion dynamics of the whole shell in 1D image and the 2D dynamics of the imploded core plasma at the same time. ${ }^{13}$ Heya et al. developed a MIXS with three arrays of pinhole images on three cathode slits to enlarge the number of identical images up to 30 and to obtain a large field of view. ${ }^{14}$

Many spectroscopic applications of MIXS have been performed after those. One is multichannel MIXS $^{15}$ which has many MIXS channels with various spectral responses. Thus it is capable of time-resolved 2D plasma temperature measurement. Another is monochromatic MIXS ${ }^{16}$ in which monochromatic images of spectral lines resolved with Bragg crystals are coupled to MIXS.

\section{B. 2D Image sampling}

Two dimensional sampling of a 2D image makes $(x, y)$ dot signals discrete both in $x$ and $y$ directions as shown in Fig. 1(c). The problem is how one can avoid overlapping of such dot signals with each other when they are streaked because the time axis and one of the space axes are the same.

In 1981, Niu et al. proposed and made a proof-ofprinciple experiment of 2D image sampling applied to an optical streak camera. ${ }^{17}$ A mask with rectangularly distributed pinholes was mounted on the square photocathode. The mask was slightly rotated to avoid overlapping of the streaked signals. A test pattern was sampled with this pinhole arrangement, streaked, and then 2D images were reconstructed from the streaked data. Although the sampling parameters were not optimized, it was an important demonstration that an areal cathode can be used as a detector plane with time-resolving capability for 2D distributed 


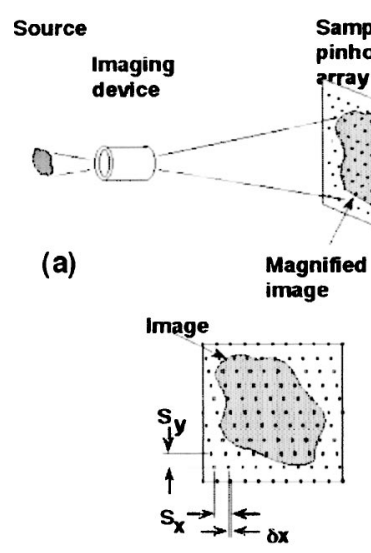

(b)

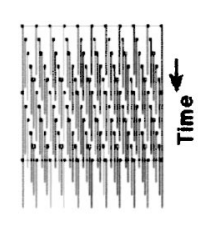

(c)

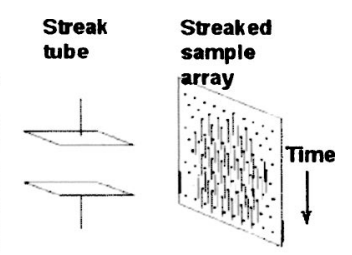

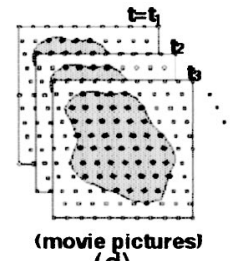

(d)
FIG. 3. Principle of 2D-SIXS imaging: (a) schematic of 2D-SIXS system, (b) 2D-distributed sampling points on the photocathode, (c) streaked data of sampling points, and (d) reconstructed time-resolved 2D images.

sampling signals. In their experiment, a framing speed of 8 $\times 10^{10}$ fps was achieved.

Two-dimensional sampling on an $\mathrm{x}$-ray streak camera was developed by Shiraga et al. in 1997 as a sampling-image $\mathrm{x}$-ray streak camera (2D-SIXS). ${ }^{7,18}$ Figure 3 shows the principle. An X-ray image is made with a single channel X-ray imager onto an areal photocathode. A disk with 2D sampling pinhole arrays is set in front of the cathode. Twodimensionally sampled signals are, then, streaked [Fig. 3(a)]. Here the sampling arrays are shifted by every line to make each recording area inbetween avoiding overlapping with sampling points in the lower lines [Figs. 3(b) and 3(c)]. Time-resolved signals of each sampling point at the corresponding time are reconstructed to form the original 2D configuration of the sampling points [Fig. 3(d) ]. A proof-ofprinciple experiment was demonstrated with $17 \times 17$ sampling points. The advantage of 2D-SIXS is that it can be coupled to a single channel x-ray microscope with very high spatial resolution (for example, $3 \mu \mathrm{m}$ with an advanced kirkpatrick-Baez

microscope, ${ }^{19}$ and $2 \mu \mathrm{m}$ with a Fresnel phase zone plate, ${ }^{20}$ while the spatial resolution of MIXS is limited by the size of the pinhole (typically $10 \mu \mathrm{m}$ ) because the pinhole is the only practical imaging device for MIXS to obtain ten or more identical images.

A similar 2D sampling applied to streak camera was developed by Kodama et al. in ultraviolet (UV) imaging ${ }^{21}$. An UV image was made on a surface of bundled fibers and the fibers were disentangled and reordered on the line in front of the photocathode slit of a UV streak camera. Time-resolved 2D images were reconstructed in a similar procedure.

It is quite interesting to see that the spatial arrangement of the sampling points in 2D-SIXS is essentially the same as that of ultrafast ISIS-CCD chips, ${ }^{22}$ in which charges from CCD pixels are stored immediately in the memory region buried between the pixels.

\section{MULTI-IMAGING X-RAY STREAK CAMERA}

As one of the most successful schemes of image sampling coupled to an x-ray streak camera, MIXS has been

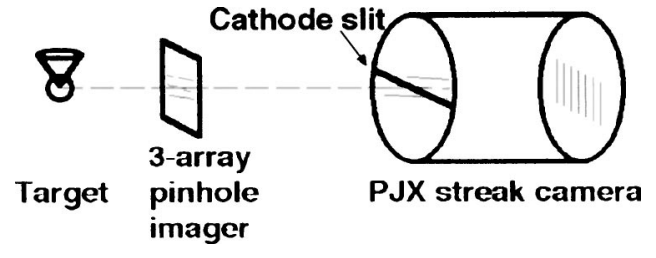

FIG. 4. Setup for MIXS measurement.

used in implosion experiments, particularly for targets related to FI. A shell target with a cone was recently proposed as a new scheme for FI, which allows direct entrance of the heating laser through the cone to the vicinity of the imploded core. Efficient heating of the core plasma in direct-drive implosion was demonstrated on the Gekko-XII laser at the Institute of Laser Engineering, Osaka University. ${ }^{10,11}$ Then, an indirect-drive implosion of a shell with a cone was investigated $^{23}$ on the OMEGA laser facility ${ }^{24}$ at the laboratory for Laser Energetics (LLE), University of Rochester. Implosion dynamics in such target are no longer in a spherical symmetry because of the existence of the cone. Thus it is of great importance to observe the dynamic behavior of the nonuniformly imploded core plasmas as well as interaction between the core and the cone.

Direct-drive implosion experiments of cone-shell targets have been performed at OMEGA laser for these purposes. The target was a plastic shell with a diameter of $870 \mu \mathrm{m}$ and a shell thickness of $25 \mu \mathrm{m}$. In some cases the shell was filled with 5-10 atm $\mathrm{D}_{2}$ or $\mathrm{D}^{3} \mathrm{He}$ gas for diagnostic use of fusion products. A cone with its half angle of $35^{\circ}$ made of gold was attached to the shell. The tip of the cone was placed at 50-100 $\mu \mathrm{m}$ from the center of the shell. The shell was irradiated with 35 beams of $0.35 \mu \mathrm{m}$ OMEGA laser except for direction of the cone. Laser energy was $15 \mathrm{~kJ}$ in a $1 \mathrm{~ns}$ square pulse. There were no heating beams in this experiment.

For MIXS measurement, a multipinhole imager was coupled to the PJX streak camera. ${ }^{25}$ Figure 4 shows the setup. The pinhole imager had three arrays of 19 pinholes with a diameter of $10 \mu \mathrm{m}$. Magnification of the imager was $12 \times$. Each pinhole array was inclined for 1D image sampling from the horizontal $(x)$ direction of the photocathode slit of the streak tube as shown in Fig. 5. Separating distances between the neighboring pinholes were $200 \mu \mathrm{m}$ in $x$ direction and $10 \mu \mathrm{m}$ and in vertical $(y)$ direction. The separation in $y(10 \mu \mathrm{m})$ was determined to give an appropriate sampling distance corresponding to the spatial resolution of the pinhole images. Nineteen images were made on the slit of the cathode. Thus the size of the field of view was $200 \mu m(x) \times 190 \mu \mathrm{m}(y)$ at the target plane. Although the

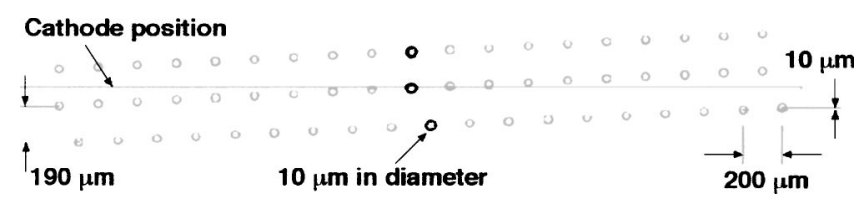

FIG. 5. Schematic of the pinhole arrays in MIXS imager. Difference in horizontal displacement between the central and upper arrays and the central and lower arrays enables us to know the direction of the misalignment in $y$ when the images are recorded in two arrays. 
initial alignment for MIXS is very tight, particularly in the $y$ direction, three arrays allowed much tolerance for misalignment of the system in $y$. Even if the system loses the central array, the upper or lower array comes into the position. The angle of the pinhole array to the cathode slit, thus the sampling separation in $y$ direction, was adjusted during the offline alignment within an accuracy of one separation $/ 19$ images $=0.5 \mu \mathrm{m}$. Pointing accuracy of the system including MIXS pointer and the ten-inch manipulator (TIM) to the target chamber center was better than $50 \mu \mathrm{m}$.

The PJX streak tube ${ }^{25}$ was designed at LLE and built by Photonis (Brive, France). The input surface is cylindrically curved with a usable photocathode length of $6 \mathrm{~cm}$. The photoelectrons are accelerated to $15 \mathrm{kV}$ through a slot electrode and focused with a quadrupole doublet. The recording system is a fiber-coupled, back-illuminated CCD camera. The system gain of $150 \mathrm{CCD}$ electrons per streak tube electron obviates the need for an image intensifer. The measured spatial resolution on the cathode is $125 \mu \mathrm{m}$ full width half maximum Gaussian and the calculated time resolution for a $1 \mathrm{~ns}$ streak is $<10 \mathrm{ps}$.

A blast shield plate (300 $\mu \mathrm{m}$ thick Be) was set in front of the pinhole disk and the photocathode material was $\mathrm{KBr}$, resulting in an observed $\mathrm{x}$-ray spectrum above $2 \mathrm{keV}$. Spatial resolutions in $x$ and in $y$ were determined by imager and cathode resolutions to be $15 \mu \mathrm{m}$ and by imager and sampling to $20 \mu \mathrm{m}$, respectively. The streaked multi-image data are sliced at a certain time and divided into a set of small 1D image pieces. Then those are rearranged to from the original 2D configuration, which is now a time-resolved image. Details of the reconstruction procedure are described in Refs. 5-7. Temporal resolution was determined by streak tube and the $2 \mathrm{D}$ reconstruction procedure (sampling width in temporal direction) to be $13 \mathrm{ps}$. When the $2 \mathrm{D}$ reconstruction is done with framing interval of $10 \mathrm{ps}, 100$ sequential frames are available from the streaked data of $1 \mathrm{~ns}$ in full scale.

The MIXS imager was mounted on PJX. After the initial off-line alignment, the while system was inserted into the OMEGA target chamber and pointed to the target chamber center with TIM.

Figure 6 is typical raw data of MIXS showing 19 streaked images. The target was one filled with $5 \mathrm{~atm} \mathrm{D}_{2}$ gas. Images in the upper half correspond to $\mathrm{x}$-ray emissions from the core and lower half to the tip of the cone. Figure 7 (a) shows 60 sequential frames reconstructed from the data shown in Fig. 6. A time-integrated image can be produced from such frames by accumulating those as shown in Fig. 7(b). Exposure time for each frame is $13 \mathrm{ps}$ and framing interval is 10 ps. Such frames can be more easily viewed when displayed as a movie on a computer screen. One can clearly see that the x-ray emission comes from the tip of the cone first, and then the imploded core is formed. The core is not moving and its shape is not spherical but like a jellyfish, with the cutout in the direction of the cone, and changing in time. From comparison with 2D simulations, it is found that the early $\mathrm{x}$-ray emission from the cone is due to shock wave propagating in the deuterium plasma inside the shell, from the center of the core towards the cone. Then the shell plasma converges and forms the core afterwards. Such pre-

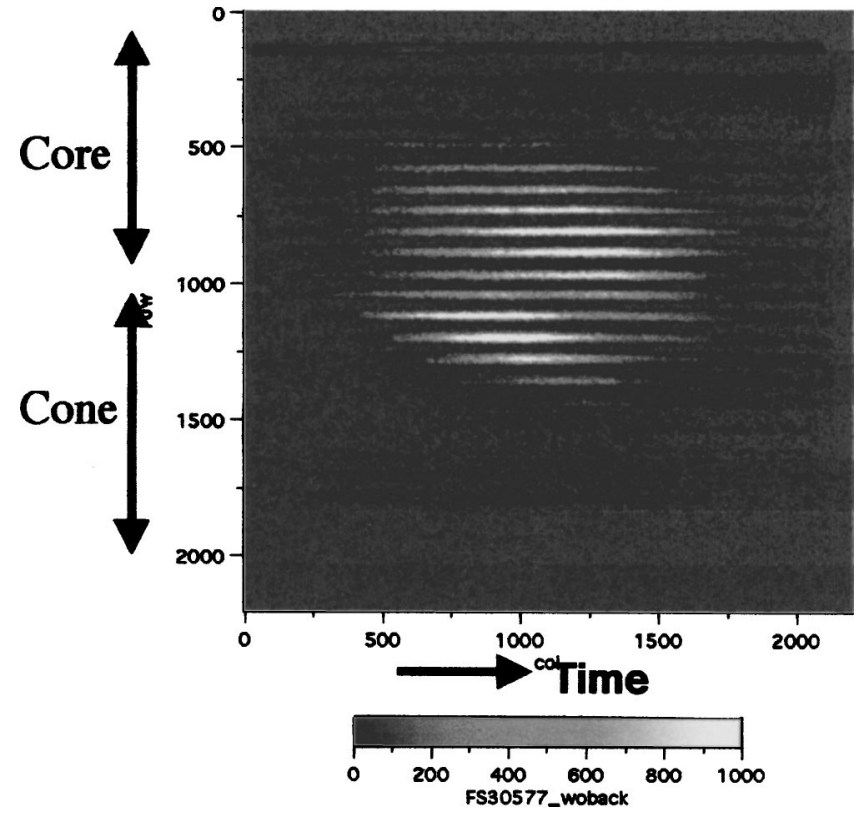

FIG. 6. Raw data of MIXS images for a gas-filled cone-shell target.

cursor shock wave towards the cone has the a possibility of degrading the cone quality before the heating beam is injected. It is of great importance to investigate such plasma dynamics in detail for FI research.

\section{DISCUSSION}

There are many advantages in MIXS: (1) Easy to obtain ultrafast 2D images; 10 ps framing is established, and even faster than $1 \mathrm{ps}$ is possible with commercially available x-ray streak cameras. (2) There is no possibility of losing information between the frames, because streaked data are continuous in time. (3) Once the image is sampled, no distortion of the image is expected to take place. (4) Spectral response of the system can be controlled by choosing filter and cathode materials. (5) Spectral applications are possible

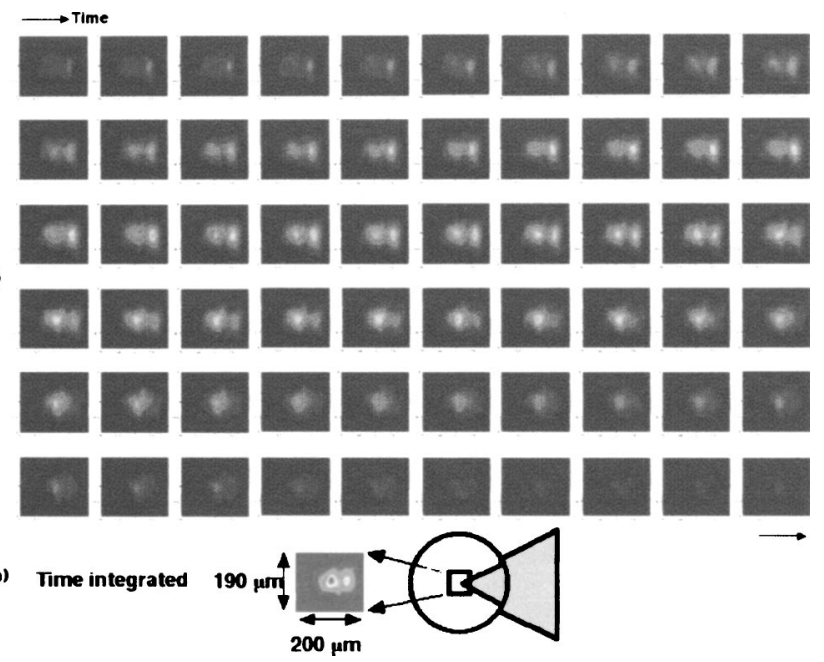

FIG. 7. 2D images reconstructed from the data shown in Fig. 6: (a) sequential frames starting from the top left, moving towards right and then down and (b) time-integrated x-ray image by accumulation of the framing data shown in (a). 
as described in Sec. I. Disadvantages are (1) Size of the viewing field is limited to relatively small values because the cathode is used in a manner divided into many pieces. (2) It is not suitable for objectives larger than the field of view. Otherwise, images are overlapped with each other on the cathode slit.

\section{ACKNOWLEDGMENTS}

The authors thank laser, target, and diagnostics crews of Gekko-XII at Osaka University and OMEGA at University of Rochester for their technical assistance. They also thank M. Heya, N. Miyanaga, T. Yamanaka, O.L. Landen, Y. Yamamoto, and G.T. Eloth for valuable discussions on image sampling, and C. Sangster and D.D. Meyerhofer for their support of the experiments at OMEGA laser facility.

${ }^{1}$ J. Nuckolls, L. Wood, A. Thiessen, and G. Zimmerman, Nature (London) 239, 139 (1972).

2 M. Katayama, M. Nakai, T. Yamanaka, Y. Izawa, and S. Nakai, Rev. Sci. Instrum. 62, 124 (1991); D. K. Bradley, P. M. Bell, J. D. Kilkenny, R. Hanks, O. L. Landen, P. A. Jaanimagi, P. W. McKenty, and C. P. Verdon, ibid. 63, 4813 (1992).

3 J. D. Kilkenny, Laser Part. Beams 9, 49 (1991); H. Azerchi et al., ibid. 9, 193 (1991); M. Katayama, H. Shiraga, M. Nakai, T. Kobayashi, and Y. Kato, Rev. Sci. Instrum. 64, 706 (1993).

${ }^{4}$ D. K. Bradley, P. M. Bell, O. L. Landen, J. D. Kilkenny, and J. Ortel, Rev. Sci. Instrum. 66, 716 (1995).

${ }^{5}$ O. L. Landen, Rev. Sci. Instrum. 63, 5075 (1992).
${ }^{6}$ H. Shiraga, M. Heya, A. Fujishima, O. Maegawa, K. Shimada, Y. Kato, T. Yamanaka, and S. Nakai, Rev. Sci. Instrum. 66, 722 (1995).

${ }^{7}$ H. Shiraga, N. Miyanaga, M. Heya, M. Nakasuji, Y. Aoki, H. Azechi, T. Yamanaka, and K. Mima, Rev. Sci. Instrum. 68, 745 (1997).

${ }^{8}$ N. G. Basov, S. Yu. Gus'kov, and L. P. Feokistov, J. Sov. Laser Res. 13, 396 (1992).

${ }^{9}$ M. Tabak, J. Hammer, M. E. Glinsky, W. L. Kruer, S. C. Wilks, J. Woodworth, E. M. Campbell, M. D. Perry, and R. J. Mason, Phys. Plasmas 1, 1626 (1994).

${ }^{10}$ R. Kodama et al., Nature (London) 412, 798 (2001).

${ }^{11} \mathrm{R}$. Kodama, et al., Nature (London) 418, 933 (2002).

${ }^{12}$ M. Sultanoff, Rev. Sci. Instrum. 21, 653 (1950).

${ }^{13}$ H. Shiraga, M. Heya, M. Nakasuji, N. Miyanaga, H. Azechi, H. Takabe, T. Yamanaka, and K. Mima, Rev. Sci. Instrum. 68, 828 (1997).

${ }^{14}$ M. Heya, S. Fujioka, H. Shiraga, N. Miyanaga, and T. Yamanaka, Rev. Sci. Instrum. 72, 755 (2001).

${ }^{15}$ M. Heya, M. Nakasuji, H. Shiraga, N. Miyanaga, H. Azechi, H. Takabe, T. Yamanaka, and K. Mima, Rev. Sci. Instrum. 68, 820 (1997).

${ }^{16}$ N. Miyanaga, Y. Aoki, H. Shiraga, K. Shimada, K. Fujimoto, M. Heya, and M. Nakasuji, Rev. Sci. Instrum. 68, 817 (1997).

${ }^{17}$ H. Niu, T. Chao, and W. Sibbett, Rev. Sci. Instrum. 52, 1190 (1981).

${ }^{18}$ H. Shiraga, M. Nakasuji, M. Heya, and N. Miyanaga, Rev. Sci. Instrum. 70, 620 (1999).

${ }^{19}$ R. Kodama, N. Ikeda, Y. Kato, Y. Katori, T. Iwai, and K. Takeshi, Opt. Lett. 21, 1321 (1996).

${ }^{20}$ Y. Tamari, Rev. Sci. Instrum these proceedings.

${ }^{21}$ R. Kodama, K. Okada, and Y. Kato, Rev. Sci. Instrum. 70, 625 (1999).

${ }^{22}$ T. G. Etoh, K. Takehara, T. Okinaka, Y. Takano, A. Ruckelshausen, and D. Poggemann, Proc. SPIE 4183, 36 (2001).

${ }^{23}$ R. Stephens, S. P. Hatchett, R. E. Turner, K. A. Tanaka, and R. Kodama, Phys. Rev. Lett. 91, 185001 (2003).

${ }^{24}$ T. R. Boehly et al., Opt. Commun. 133, 495 (1997).

${ }^{25}$ P. Jaanimagi (unpublished). 